\section{TECHNICAL NOTE}

\section{T. Schneider}

J. Hom

J. Bredno

J.W. Dankbaar

S.-C. Cheng

M. Wintermark

\title{
Delay Correction for the Assessment of Blood- Brain Barrier Permeability Using First-Pass Dynamic Perfusion CT
}

SUMMARY: Hemorrhagic transformation is a serious potential complication of ischemic stroke with damage to the BBB as one of the contributing mechanisms. BBB permeability measurements extracted from PCT by using the Patlak model can provide a valuable assessment of the extent of BBB damage. Unfortunately, Patlak assumptions require extended PCT acquisition, increasing the risk of motion artifacts. A necessary correction is presented for obtaining accurate BBB permeability measurements from first-pass PCT.

ABBREVIATIONS: $\mathrm{ACA}=$ anterior cerebral artery; $\mathrm{AIF}=$ arterial input function; $\mathrm{BBB}=$ blood-brain barrier; $\mathrm{BBBP}=$ blood-brain barrier permeability; $\mathrm{CBF}=$ cerebral blood flow; $\mathrm{CBV}=$ cerebral blood volume; $\mathrm{Cl}=$ confidence interval; $\mathrm{CTA}=\mathrm{CT}$ angiography; FLAIR = fluid-attenuated inversion recovery; $\mathrm{GEE}=$ generalized estimating equations; $\mathrm{MCA}=$ middle cerebral artery; $\sqrt{\mathrm{MSE}}=$ root mean squared errors; MSE = mean squared error; MTT = mean transit time; $\mathrm{NCCT}=$ noncontrast $\mathrm{CT}$; $\mathrm{PCA}=$ posterior cerebral artery; $\mathrm{PCT}=$ perfusion $\mathrm{CT}$; $\mathrm{PET}=$ positron-emission tomography; $\mathrm{RIF}=$ region input function; rtPA $=$ recombinant tissue plasminogen activator; TTP $=$ time to peak

H emorrhagic transformation is a serious and potentially fatal complication of ischemic stroke. ${ }^{1}$ The risk of this complication has been and is a hindrance to the administration of rtPA for the treatment of this condition. ${ }^{2,3}$ Disruption of the BBB is believed to be one of the factors involved in the pathophysiology of hemorrhagic transformation. ${ }^{4}$ It is hypothesized that the identification of a disrupted BBB in patients with stroke before rtPA administration would be a valuable tool in assessing the risks/benefits of this treatment. ${ }^{5}$

PCT has been used for some time to assess cerebral hemodynamics. ${ }^{6}$ Recently, measurements of BBBP have also been extracted from PCT data, ${ }^{7}$ with the Patlak model ${ }^{8}$ one of the methods used. Earlier studies with this model used PCT data collected from the first-pass of contrast injection. ${ }^{7,9}$ However, the original Patlak description clearly states that a steady-state of contrast levels must be obtained before BBBP can be properly assessed. ${ }^{8}$ A recent study ${ }^{10}$ demonstrated that acquiring a delayed PCT acquisition was required to accurately assess BBBP according to the Patlak model. This delayed acquisition

Received December 26, 2009; accepted after revision March 23, 2010.

From the Department of Radiology, Neuroradiology Section (T.S., J.H., J.W.D., M.W.), and Department of Epidemiology and Biostatistics (S.-C.C.), University of California, San Francisco, San Francisco, California; Clinical Sites Research Program (J.B.), CT Clinical Science Group and Philips Research North America, Philips Healthcare, Briarcliff Manor, New York; and Department of Radiology (J.W.D.), University Medical Center, Utrecht, the Netherlands.

Max Wintermark receives funding from the National Center for Research Resources, grant KL2 RR024130, Philips Healthcare, and GE Healthcare. Joerg Bredno is an employee of Philips Healthcare.

The content of the article is solely the responsibility of the authors and does not necessarily represent the official views of the National Institute of Neurologic Disorders and Stroke, the National Center for Research Resources, the National Institutes of Health, or the other sponsors.

Please address correspondence to Max Wintermark, MD, MAS, Department of Radiology, Division of Neuroradiology, University of Virginia Health System, P.0. Box 800170, Charlottesville, VA, 22908-0170; e-mail: mw4v@virginia.edu

Indicates open access to non-subscribers at www.ajnr.org

DOI 10.3174/ajnr.A2152 needs to be at least 210 seconds. ${ }^{11}$ This relatively long scanning duration raises issues of motion artifacts and radiation dose. Additional approaches to solve these problems are warranted. In this report, we present a further correction to the current Patlak model and test its validity on a sample of PCT images of patients with ischemic stroke.

\section{Technique}

\section{Theory}

Patlak et $\mathrm{al}^{8}$ and Patlak and Blasberg ${ }^{8,12}$ described a theoretic model of blood-brain exchange. It assumes the unidirectional transfer of a tracer from a reversible (arterial) compartment to an irreversible extravascular space (in this case the brain parenchyma) for a certain period of time. Transfer of tracer is assumed to be unidirectional when a steady-state phase is reached between intravascular and extravascular compartments. Simulated brain PCT studies ${ }^{13}$ were used to help validate 2 major assumptions, no backflow and leakage of BBB as early as the first pass.

The graphic representation of the Patlak model is called the Patlak plot. When the Patlak plot is linear, unidirectional transfer is said to be present. The slope of the plot indicates the rate of transfer between the 2 compartments. When applied to PCT, the Patlak model uses iodinated contrast as the tracer and takes advantage of the Hounsfield attenuation being directly proportional to the iodinated contrast concentration.

The derivation of the Patlak plot comes from the idea that the total attenuated contrast of a current voxel or region of interest can be represented as a sum of the tracer in the intravascular and extravascular compartments at a specified time $t$ :

$$
\operatorname{RIF}(t)=\mathrm{CBV} \cdot c_{\mathrm{iv}}(t)+K_{1} \int_{0}^{t} c_{\mathrm{iv}}(\tau) d \tau,
$$

$$
\text { Intravascular }=\mathrm{CBV} \cdot c_{\mathrm{iv}}(t) \text {, }
$$

$$
\text { Extravascular }=K_{1} \int_{0}^{t} c_{\text {iv }}(\tau) d \tau
$$


where $\operatorname{RIF}(t)$ is the region input function or contrast at time $t$, and $c_{\mathrm{iv}}(t)$ is the intravascular concentration of the voxel at time $t$. Multiplying the 2 would yield the total contrast residing in the intravascular component. $\int_{0}^{t} c_{i v}(\tau) d \tau$ represents the total amount of contrast that perfused the intravascular component from time 0 up until time $t$, and $K_{1}$ is the permeability constant, which is the result of the Patlak analysis. Because the amount of contrast that leaks from the intravascular to the extravascular component is directly related to the permeability, $K_{1}$ is multiplied by $\int_{0}^{t} c_{i v}(\tau) d \tau$.

Of course, the intravascular and extravascular components of each CT voxel are imaged together, which results in an unknown true intravascular concentration. The $A I F$ or $A I F(t)$ is used instead because the voxel in that region contains only an intravascular component. The equation now becomes

$$
\operatorname{RIF}(t)=\mathrm{CBV} \cdot A I F(t)+K_{1} \int_{0}^{t} A I F(\tau) d \tau,
$$

The Patlak model takes this equation, divides it by $\operatorname{AIF}(t)$, which yields an easily plotable line in which the slope is $K_{1}$ :

3) $\frac{\operatorname{RIF}(t)}{A I F(t)}=\mathrm{CBV}+K_{1} \frac{\int_{0}^{t} A I F(\tau) d \tau}{A I F(t)}$

$$
\begin{gathered}
\text { setting } Y=\frac{\operatorname{RIF}(t)}{A I F(t)} \text { and } X=\frac{\int_{0}^{t} A I F(\tau) d \tau}{A I F(t)} \text { yields } \\
Y=\mathrm{CBV}+K_{1} \cdot X .
\end{gathered}
$$

The end result of the Patlak model is shown in equation 3, which is a linear regression from the PCT datasets. The slope $K_{1}$ is used as an indicator of BBBP. The extravascular component is simplified by the fact that it does not take into account the backflow of contrast. A major reason for its removal is that backflow heavily complicates the analysis in which only nonlinear regression techniques may be used. This simplicity of the Patlak model allows efficient creation of visual linear graph plots and functional maps of $K_{1}$. Furthermore, simulation studies ${ }^{13}$ have shown that backflow is a negligible component of permeability in cases of stroke, even in the delayed-acquisition stages.

Again, the Patlak model requires a steady-state phase to be reached between compartments so that the transfer of tracer can be assumed to be unidirectional. A steady-state phase can only occur after the initial rapid changes in tracer concentration have subsided so that the arterial concentration has stabilized, allowing a constant rate of transfer of contrast. The replacement of $\mathrm{c}_{\mathrm{iv}}(t)$ with $A I F(t)$ can become a significant problem if the arterial function curve is ahead of the current voxel. This is because the voxel with a delayed arrival of contrast will have an incorrectly lower $\operatorname{RIF}(t)$ at the beginning of the first pass for the artery (not the region of interest) because contrast has yet to arrive in the region of interest and there is an incorrectly higher $\operatorname{RIF}(t)$ at the end of the first pass for the artery because contrast has yet to leave the region of interest. This will lead to an increase of the slope, which is ultimately the reason why BBBP measurements extracted from first-pass PCT data by using the Patlak model tend to be overestimated. ${ }^{10}$ This phenomenon is most important in ischemic regions because there the flow is significantly delayed compared with other regions. ${ }^{10}$

In the method we propose for obtaining accurate BBBP measurements from first-pass PCT, we correct time-enhancement curves for delay. More specifically, the time-enhancement curve in each parenchymal voxel is shifted so that the arrival of contrast occurs at the same time as the arterial input curve:

4)

$$
c_{\mathrm{iv}}(t)=\operatorname{AIF}(t-[T T P(\mathrm{RIF})-\operatorname{TTP}(\mathrm{AIF})]) .
$$

TTP is the time till the peak of a curve. From previous simulation studies, TTP was shown to be the best estimator of the true delay of contrast. ${ }^{13}$ The new estimate $c_{\text {iv }}(t)$ is then put back into equation 3 in place of the $\operatorname{AIF}(t)$ for all delay-corrected calculations.

\section{Validation Study Design}

Imaging data obtained as part of standard clinical stroke care at our institution were retrospectively reviewed with the approval of the institutional review board. At our institution, patients with suspicion of acute stroke and no history of significant renal insufficiency or contrast allergy routinely undergo a stroke CT survey including NCCT of the brain, PCT at 2 cross-sectional positions, CTA of the cervical and intracranial vessels, and postcontrast cerebral CT, obtained in this chronologic sequence.

We retrospectively identified a consecutive series of 23 patients admitted from July 2007 to August 2008 who met the following inclusion criteria: 1) admission to the emergency department with signs and symptoms suggesting hemispheric stroke within 12 hours after symptom onset, 2) documentation of acute ischemic hemispheric stroke by both admission stroke protocol and clinical examination, 3) no evidence of intracerebral hemorrhage on the admission NCCT, and 4) no significant motion artifacts following application of the registration algorithm. Patients' demographic and clinical data were recorded.

\section{Imaging Protocol}

PCT studies were obtained on 64-section CT scanners. Each PCT study involved successive gantry rotations performed in cine mode during intravenous administration of iodinated contrast material. Images were acquired and reconstructed at a temporal sampling rate of 1 image per second for the first 37 seconds and 1 image every 2 seconds for the next 33 seconds. Additional gantry rotations were obtained at 90, 120, 150, 180, 210, and 240 seconds. Acquisition parameters were $80 \mathrm{kV}$ (peak) and 100 mAs. Two successive PCT series at 2 different levels were performed following the NCCT and before the CTA. At each PCT level, eight 5-mmthick sections were assessed. The first PCT series was obtained at the level of the third ventricle and the basal ganglia, and the second PCT series, above the lateral ventricles. For each PCT series, a $40-\mathrm{mL}$ bolus of iohexol (Omnipaque; Amersham Health, Princeton, New Jersey; 300 mg/mL of iodine) was administered into an antecubital vein by using a power injector at an injection rate of $5 \mathrm{~mL}$ per second for all patients. CT scanning was initiated 7 seconds after the start of the injection of the contrast bolus. Data from both boluses were used because prior work has demonstrated that there is no significant parenchymal saturation effect from the first bolus that leads to underestimation of BBBP from data from the second bolus. $^{14}$

\section{Image Postprocessing}

PCT data were analyzed using PCT software developed by Philips Medical Systems (Cleveland, Ohio). This software relies on the 


\begin{tabular}{lc}
\hline \multicolumn{2}{l}{ Table 1: Study patient characteristics } \\
\hline Patients with Stroke \\
\hline No. of patients & 23 \\
No. of men (\%) & $8(35 \%)$ \\
Age (yr) & Median $=74$ \\
& Interquartile range $=59.5-82.5$ \\
Time from stroke to PCT (hr) & Range $=26-92$ \\
& Median $=2.25$ \\
Stroke location: & Interquartile range $=1.5-5$ \\
ACA and MCA territories & Range $=1-11.75$ \\
MCA territory & \\
ACA territory & 3 \\
PCA territory & 20 \\
\hline
\end{tabular}

central-volume principle, which is the most accurate for lowinjection rates of iodinated contrast material. ${ }^{15}$ The software obtains mathematic descriptions of the time-attenuation curves for each pixel, by applying curve fitting by least mean squares, after correcting for motion and noise reduction through an anisotropic edge-preserving spatial filter. A closed-form (noniterative) deconvolution is then applied to calculate the MTT map. ${ }^{16}$ The deconvolution operation requires a reference AIF (most often within the ACA), automatically selected by the PCT software within a region of interest drawn by the user. The CBV map is calculated from the area under the time-attenuation curves, ${ }^{17}$ not from the Patlak model $^{8}$ used to calculate BBBP, because the CBV deconvolution method can use all gantry rotations for its calculation, be less susceptible to noise, and provide the BBBP calculations with greater stability. The PCT infarct core and salvageable brain tissue regions of interest are automatically calculated by the software by using CBV thresholds and MTT thresholds reported in the literature as the most accurate (PCT salvageable brain tissue: MTT $>$ $145 \%$ of the contralateral side values plus $\mathrm{CBV} \geq 2.0 \mathrm{~mL} \times 100$ $\mathrm{g}^{-1}$; PCT infarct core: MTT $>145 \%$ of the contralateral side values plus $\left.\mathrm{CBV}<2.0 \mathrm{~mL} \times 100 \mathrm{~g}^{-1}\right) .{ }^{18}$ Regions of interest for each patient were thus divided into regions of interest of tissue at risk, infarct, and normal parenchyma according to these thresholds.

BBBP measurements were extracted from PCT data by using a second prototype software developed by Philips Medical Systems. This software is based on the Patlak model. ${ }^{8}$ For this study, 2 types of BBBP values were calculated: one used delay correction, and the other did not.

\section{Data Analysis}

Using the prototype software, we calculated BBBP values from the reference standard delayed-acquisition dataset, which incorporated all time points from 90 to 240 seconds. Additionally, we calculated BBBP values by using the delay-corrected Patlak model by using the first-pass 0 - to 90-second PCT data and also by using the first-pass 30 to 90 -second PCT data. To quantify the effect of the delay correction, we also calculated BBBP values by using no delay correction from the first-pass 0- to 90-second PCT data. BBBP values were recorded in the infarct and tissue at risk, as automatically delineated by the software, as well as in mirrored regions of interest in the contralateral nonischemic tissue (also automatically delineated by the software).

We quantified the quality of the linear fit to see how well the assumptions of the Patlak model were met by data extracted from the reference standard 90- to 240-second acquisition (no delay correction needed), the 0 - to 90 -second first-pass acquisition with delay correction, the 30 - to 90-second first-pass acquisition with delay correction, and the 0 - to 90second first-pass acquisition without delay correction. To do this, we used the $\sqrt{ }$ MSE, which is a measure of variability of data points around a straight line: A value close to 0 indicates a smaller spread of data points around the line, corresponding to a better fit.

\section{Statistical Analysis}

The 4 sets of calculated BBBP values (reference standard, 90- to 240second acquisition, 0 - to 90 -second first-pass acquisition with delay correction, 30- to 90-second first-pass acquisition with delay correction, and 0 - to 90 -second first-pass acquisition without delay correction) as well as the corresponding $\sqrt{ }$ MSE values for the ischemic and nonischemic regions of interest were compared by using GEE models with a robust variance estimation, with fixed effects for patients and type of regions of interest. Because the distribution of the parameters was not normal but skewed, rather than reporting simple means, we described estimated mean values (obtained by log transformation of the data). For all values, 95\% CIs were also calculated.

\section{Results}

\section{Patients and Imaging Studies}

Twenty-three patients who matched our inclusion criteria were retrospectively identified. Patient characteristics are summarized in Table 1 . The median time from symptom onset to PCT was 2.25 hours (range, $1-11.75$ hours). The Patlak analyses were performed in a total number of 230 ischemic regions of interest and 230 nonischemic regions of interest.

\section{BBBP Measurements for Delayed PCT Acquisition}

BBBP values calculated from the delay-corrected 30- to 90second first-pass acquisition overlapped with the ones calculated from the reference standard 90- to 240-second delayed acquisition in the ischemic parenchymal tissue and were slightly underestimated in the nonischemic parenchymal tissue (Table 2, Figs 1 and 2). The delay-corrected first pass did not show the overestimation of BBBP values in the tissue at

\begin{tabular}{|c|c|c|c|c|}
\hline & 90-240 Seconds & 30-90 Seconds & 0-90 Seconds & 0-90 Seconds \\
\hline & $\begin{array}{l}\text { Reference } \\
\text { Standard }\end{array}$ & $\begin{array}{c}\text { Delay } \\
\text { Correction }\end{array}$ & $\begin{array}{c}\text { Delay } \\
\text { Correction }\end{array}$ & $\begin{array}{l}\text { No Delay } \\
\text { Correction }\end{array}$ \\
\hline Nonischemic & $2.07(1.83-2.34)$ & $\begin{array}{c}1.54(1.39-1.71) \\
P<.001\end{array}$ & $\begin{array}{c}1.57(1.24-2.00) \\
P=.002\end{array}$ & $\begin{array}{c}1.95(1.74-2.18) \\
P=.125\end{array}$ \\
\hline Infarct & $2.48(2.15-2.87)$ & $\begin{array}{c}2.09(1.85-2.37) \\
P=.074\end{array}$ & $\begin{array}{c}1.66(1.30-2.11) \\
P<.001\end{array}$ & $\begin{array}{c}2.49(2.21-2.79) \\
P=.874\end{array}$ \\
\hline Tissue at risk & $2.43(2.16-2.74)$ & $\begin{array}{c}2.45(2.21-2.72) \\
P=.921\end{array}$ & $\begin{array}{c}1.93(1.52-2.46) \\
P=.066\end{array}$ & $\begin{array}{c}3.49(3.14-3.88) \\
P<.001\end{array}$ \\
\hline
\end{tabular}

${ }^{a}$ Mean absolute permeability values and corresponding 95\% Cls shown for different regions-of-interest and acquisition-time datasets, as well as $P$ values derived from the GEE models. 


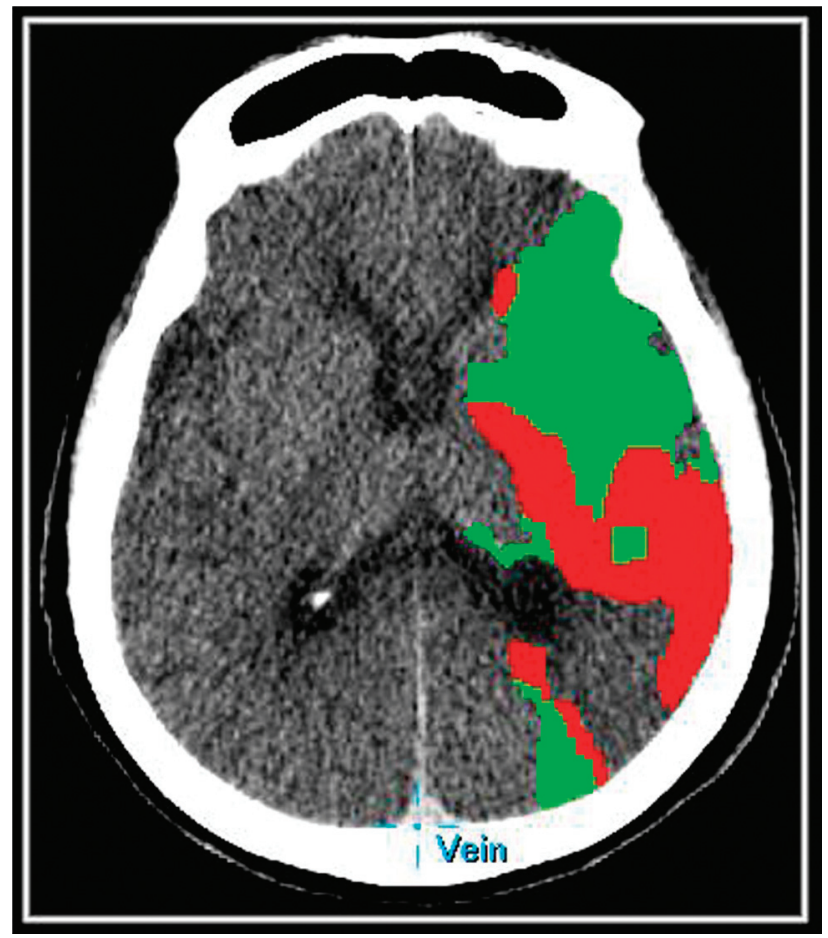

Fig 1. A 75-year-old patient with a left MCA ischemic stroke. Prognostic PCT map shows a mixed pattern of infarct core (red) and penumbra (green). risk that was seen with the first-pass acquisition without delay correction (Table 2).

Quality of the Linear Regression According to the Patlak Model

$\checkmark$ MSE measurements (Table 3) were low for the reference standard 90- to 240-second delayed acquisition and for the delay-corrected 30- to 90-second first-pass acquisition, showing that the linearity assumption underlying the Patlak model is well-respected. For the first-pass acquisition without delay correction, $\sqrt{ }$ MSE measurements were higher, indicating a poorer linear fitting.

\section{Radiation Dose and Motion Artifacts}

The effective radiation dose associated with the reference standard 240-second acquisition was calculated (3.2 mSv). The decrease in radiation dose associated with a transition to a 90 -second first-pass-only acquisition was $8.3 \%$.

Motion artifacts significant enough to interfere with permeability processing even after application of the registration algorithm were seen in 3 patients. In each of these cases, suitable permeability processing was not possible with the full 90to 240-second acquisition. However, in 2 of these patients, BBBP values could be calculated from the 90 -second first-pass acquisition because the motion artifacts affected exclusively the delayed acquisition (but spared the first-pass).

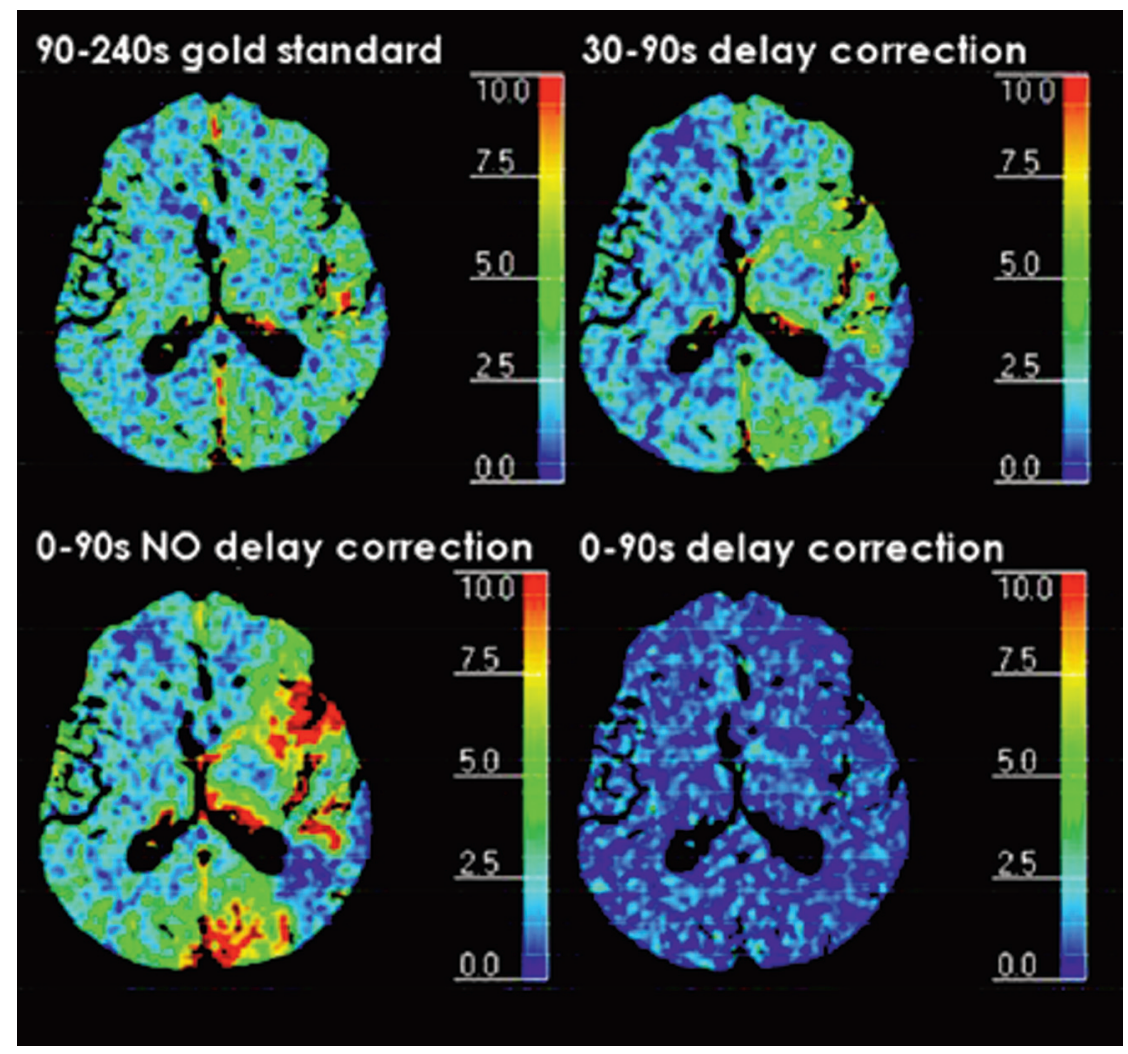

Fig 2. Absolute permeability (milliliters per $100 \mathrm{~g}$ per minute) maps for the reference standard 90 - to 240 -second delayed acquisition and for the 0 - to 90 -second first-pass acquisition with delay correction, the 30- to 90-second first-pass acquisition with delay correction, and the 0 - to 90 -second first-pass acquisition without delay correction. BBBP values calculated from the delay-corrected 30- to 90-second first-pass acquisition overlapped with the ones calculated from the reference standard 90- to 240-second delayed acquisition in the ischemic parenchymal tissue and were slightly underestimated in the nonischemic parenchymal tissue. The delay-corrected first-pass did not show the overestimation of BBBP values in the penumbra that were seen with the first-pass acquisition without delay correction. 
Table 3: $\sqrt{ }$ MSE calculated for the fitting of the Patlak line ${ }^{a}$

\begin{tabular}{|c|c|c|c|c|}
\hline & 90-240 Seconds & 30-90 Seconds & 0-90 Seconds & 0-90 Seconds \\
\hline & $\begin{array}{l}\text { Reference } \\
\text { Standard }\end{array}$ & $\begin{array}{c}\text { Delay } \\
\text { Correction }\end{array}$ & $\begin{array}{c}\text { Delay } \\
\text { Correction }\end{array}$ & $\begin{array}{l}\text { No Delay } \\
\text { Correction }\end{array}$ \\
\hline Nonischemic & $1.56(1.36,1.79)$ & $\begin{array}{c}1.12(0.97-1.30) \\
P<.001\end{array}$ & $\begin{array}{c}2.38(1.74-3.26) \\
P=.002\end{array}$ & $\begin{array}{c}4.04(3.04-5.36) \\
P<.001\end{array}$ \\
\hline Infarct & $1.79(1.49,2.15)$ & $\begin{array}{c}1.19(1.02-1.39) \\
P<.001\end{array}$ & $\begin{array}{c}2.54(1.85-3.49) \\
P=.026\end{array}$ & $\begin{array}{c}4.05(3.03-5.38) \\
P<.001\end{array}$ \\
\hline Tissue at risk & $1.65(1.45,1.87)$ & $\begin{array}{c}1.14(1.00-1.29) \\
P<.001\end{array}$ & $\begin{array}{c}2.86(2.07-3.95) \\
P<.001\end{array}$ & $\begin{array}{c}4.25(3.24-5.56) \\
P<.001\end{array}$ \\
\hline
\end{tabular}

${ }^{a}$ Mean $\sqrt{ }$ MSE and corresponding $95 \%$ Cls are shown for different region-of-interest and acquisition-time datasets, as well as $P$ values derived from the GEE models. $\sqrt{ }$ MSE is a measure of variability of data points around a straight line: a value close to zero indicates a smaller spread of data points around the line, corresponding to a better fit.

\section{Discussion}

The original description of the Patlak model, published in 1983, contains a very stringent assumption that steady-state has been achieved. ${ }^{8}$ This assumption requires a delayed acquisition. There would be advantages to applying the Patlak model to first-pass PCT data because this is usually what is obtained as part of standard PCT protocols. However, recent results show that this results in overestimation of permeability values, mainly in the tissue at risk because of the delayed contrast arrival. ${ }^{10}$ To overcome this pitfall, we have developed an additional modification of the delay-corrected Patlak model for the first-pass PCT data.

Our results are promising and show that BBBP values calculated from the delay-corrected, 30- to 90-second first-pass acquisition overlapped with the ones calculated from the reference standard 90- to 240-second delayed acquisition in the ischemic parenchymal tissue and were slightly underestimated in the nonischemic parenchymal tissue. The delay-corrected first-pass did not show the significant overestimation of BBBP values in the tissue at risk that was seen with the first-pass acquisition without delay correction. Also, the linearity assumption underlying the Patlak model is well-respected by the delay-corrected first-pass. Looking at the absolute permeability values (Table 2 ), we did not see very large differences between the reference standard, delay correction, and no delay correction. However, the $\sqrt{ }$ MSEs (Table 3) were significantly higher for no delay correction compared with the reference standard and delay correction. This finding indicates that delay correction leads to more stable and consistent results for shorter acquisitions. The small differences in terms of absolute permeability values between delay-corrected short acquisition and reference standard-delayed acquisition need to be tested in terms of clinical relevance in future studies.

Despite our effort to correct for the delayed arrival time of contrast, the time interval 30-90 seconds still worked better than the $0-90$-second time interval. The simplest explanation for this is that even with the correction, the time points $0-30$ seconds are still not steady enough to be used in the Patlak model. It is only near the end of the first pass that the values can level out enough to create an environment suitable for this model.

In terms of limitations of our study, we focused on the Patlak model, and we did not assess other non-Patlak models that can be applied to PCT data to calculate BBBP, such as the distributed parameter models. ${ }^{19,20}$ Future studies are needed to determine whether our conclusions hold true for alternative models.

\section{Conclusions}

In conclusion, we developed a modified version of the Patlak model with delay correction that allowed obtaining nonover- estimated BBBP values from a standard first-pass PCT acquisition, minimizing the risk of motion artifacts associated with a prolonged acquisition.

\section{References}

1. Alexandrov AV, Black SE, Ehrlich LE, et al. Predictors of hemorrhagic transformation occurring spontaneously and on anticoagulants in patients with acute ischemic stroke. Stroke 1997;28:1198-202

2. Jaillard A, Cornu C, Durieux A, et al. Hemorrhagic transformation in acute ischemic stroke: The MAST-E study-MAST-E Group. Stroke 1999; 30:1326-32

3. Su EJ, Fredriksson L, Geyer M, et al. Activation of PDGF-CC by tissue plasminogen activator impairs blood-brain barrier integrity during ischemic stroke. Nat Med 2008;14:731-37. Epub 2008 Jun 22

4. Wang X, Lo EH. Triggers and mediators of hemorrhagic transformation in cerebral ischemia. Mol Neurobiol 2003;28:229-44

5. Bisdas S, Hartel M, Cheong LH, et al. Prediction of subsequent hemorrhage in acute ischemic stroke using permeability CT imaging and a distributed parameter tracer kinetic model. J Neuroradiol 2007;34:101-08

6. Lee T-Y. Functional CT: physiological models. Trends in Biotechnology 2002;20:S3-S10

7. Cianfoni A, Cha S, Bradley WG, et al. Quantitative measurement of bloodbrain-barrier permeability using perfusion-CT in extra-axial brain tumors. J Neuroradiol 2006;33:164-68

8. Patlak CS, Blasberg RG, Fenstermacher JD. Graphical evaluation of blood-tobrain transfer constants from multiple-time uptake data. J CerebBlood Flow Metab 1983;3:1-7

9. Lin K, Kazmi KS, Law M, et al. Measuring elevated microvascular permeability and predicting hemorrhagic transformation in acute ischemic stroke using first-pass dynamic perfusion CT imaging. AJNR Am J Neuroradiol 2007;28:1292-98

10. Dankbaar JW, Hom J, Schneider T, et al. Dynamic perfusion CT assessment of the blood-brain barrier permeability: first pass versus delayed acquisition. AJNR Am J Neuroradiol 2008;29:1671-76

11. Hom J, Dankbaar JW, Schneider T, et al. Optimal duration of acquisition for dynamic perfusion CT assessment of blood-brain barrier permeability using the Patlak model. AJNR Am J Neuroradiol 2009;30:1366-70. Epub 2009 Apr 15

12. Patlak CS, Blasberg RG. Graphical evaluation of blood-to-brain transfer constants from multiple-time uptake data: generalizations. J Cereb Blood Flow Metab 1985;5:584-90

13. Bredno J, Hom J, Schneider T, et al. Simulation-based validation and arrivaltime correction for Patlak analyses of perfusion-CT scans. Proc Soc Photo Opt Instrum Eng 2009;72620G

14. Dankbaar JW, Hom J, Schneider T, et al. Accuracy and anatomical coverage of perfusion CT assessment of the blood-brain barrier permeability: one bolus versus two boluses. Cerebrovasc Dis 2008;26:600-05

15. Wintermark M, Maeder $P$, Thiran JP, et al. Quantitative assessment of regional cerebral blood flows by perfusion CT studies at low injection rates: a critical review of the underlying theoretical models. Eur Radiol 2001;11:1220-30

16. Axel L. Tissue mean transit time from dynamic computed tomography by a simple deconvolution technique. Invest Radiol 1983;18:94-99

17. Ladurner G, Zilkha E, Iliff D, et al. Measurement of regional cerebral blood volume by computerized axial tomography. J Neurol Neurosurg Psychiatry 1976;39:152-58

18. Wintermark M, Flanders AE, Velthuis B, et al. Perfusion-CT assessment of infarct core and penumbra: receiver operating characteristic curve analysis in 130 patients suspected of acute hemispheric stroke. Stroke 2006;37:979-85

19. Bisdas S, Hartel M, Cheong LH, et al. Detection of early vessel leakiness in acute ischemic stroke using computed tomography perfusion may indicate hemorrhagic transformation. Acta Radiol 2007;48:341-44

20. Johnson JA, Wilson TA. A model for capillary exchange. Am J Physiol 1966;210: $1299-303$ 